\title{
Special section on software cybernetics
}

\author{
Rene Boel
}

Published online: 29 February 2012

(C) Springer Science+Business Media, LLC 2012

This special section on software cybernetics deals with novel results and new methodologies that bring together software engineering and discrete event control theory ideas. Papers treating distinct issues and techniques regarding software engineering, such as component based and secure development, using various control theory approaches, relevant to the interplay between software engineering and control, were solicited.

After careful reviewing the following paper was selected from a large list of papers under consideration:

Changyan Zhou and Ratnesh Kumar: Semantic Translation of Simulink Diagrams to Input/Output Extended Finite Automata

We would like to thank the editors of this special section, Joao Cangusu, Ron Kennett, and Ratnesh Kumar, for their efforts in putting together this special section.

R. Boel (凶)

EESA-SYSTeMS, University of Ghent, Tehnologiepark 914, Zwijnaarde 9052, Belgium

e-mail: rene.boel@ugent.be 